\title{
A Stochastic Memory Model for ADL Detection in Human Households ${ }^{\dagger}$
}

\author{
Jana Clement * (i) and Klaus Kabitzsch \\ Department of Computer Science, University of Technology Dresden, 01062 Dresden, Germany; \\ klaus.kabitzsch@tu-dresden.de \\ * Correspondence: jana.clement@tu-dresden.de; Tel.: +49-351-4633-8375 \\ + This paper is an extended version of our paper published in the 10th International Conference on Pervasive \\ Technologies Related to Assistive Environments (PETRA), Island of Rhodes, Greece, 21-23 June 2017.
}

Received: 16 October 2017; Accepted: 24 November 2017; Published: 30 November 2017

\begin{abstract}
Many Human Activity Recognition (HAR) systems are able to detect sequential executed Activity of Daily Living (ADL). However, a person is capable of doing two things in parallel or pausing one ADL and finishing it later. Thus, a HAR system must be capable of remembering and deciding which ADL is completed and which might be continued after the current ADL. We address this case by combining a stochastic Markov model and a psychological memory function to detect parallel ADL. For the evaluation, we use an input dataset and a publicly available benchmark. Our approach outperforms the leading HAR systems for the used benchmark by $5 \%$, while using a more cost-effective installation environment. Furthermore, we address an unsupervised learning method to train the HAR system and explain the algorithm of parallel ADL detection in detail.
\end{abstract}

Keywords: Markov model; ADL; human activity detection; cognitive model; Ambient Assisted Living (AAL); human behavior; HAR

\section{Introduction}

This paper is an extended version of the publication done in 2017 for the Pervasive Technologies Related to Assistive Environments (PETRA) Conference in Greece [1]. Additional information and figures have been added and discussed.

The detection of activities in private households becomes more important for health-care and assisted accommodations because impaired people want to continue living in their homes [2], which leads to an increase of care. Many accidents happen at home, most of them in the bathroom, living room and the kitchen, where $75 \%$ were falls [3]. Especially elderly people are afraid of accidents at home. In Germany, 34.5\% of emergency calls have no emergency reason, and $42 \%$ of them are executed by people over 70 years [4]. The fear of life-threating situations rises with people's age and so the emergency costs that could have been prevented. The elderly also tend to forget started activities, such as cooking or an open water tap. This forgetfulness may lead to a severe threat to health. Studies revealed that unattended cooking is the main leading factor responsible for fires in the kitchen $[5,6]$. Additionally, even if the person does not suffer any health-threatening consequences, there might still be considerable damage to the house's infrastructure. In [2], a study revealed that, "All men and women expressed wanting to stay at home as long as possible, even if a home care assistant was needed." The work in [2] also reveals that "Perceiving a situation of being in need of extensive care and living at a nursing home was connected to many different kinds of feelings and fears". Additionally, already physically impaired people want to be monitored due to the fear of deterioration being left unnoticed [7]. Thus, Human Activity Recognition (HAR) systems can help to prolong the elderly person's stay at home. It relaxes relatives who are concerned about the health of their family members 
by having feedback on their relative's activities. Additionally, HAR systems can also help caregivers to manage their work more efficiently.

Whereas many sensors currently used for HAR systems try to offer an appeasing design, they still are visible and may trigger fear of surveillance. In our approach, we make use of already installed, unobtrusive sensors like for example smart meters or smart plugs. An additional benefit of this practice is the reduced costs for sensors, installation and services. Most of the research models for HAR are trained with a labeled training set [8-11]. That means the monitored person has to give feedback about his/her activities. This is a very time-consuming and error-prone procedure. We already experienced this in two projects $[11,12]$ and explain it in detail later. This paper introduces an unsupervised training algorithm based on general assumptions matching all private households where no prior manual logging of activities is needed. The next section provides an overview of the state of the art in HAR. Section 3 explains how a Markov model is used to learn human activity patterns followed by a section explaining the calculation of significant factors for human patterns. A model for human memory capacity is introduced in Section 5 to calculate a sensor distribution function in Section 6 . That will be used for activity recognition in Section 7. Afterwards, an evaluation is done, and the conclusion will be drawn.

\section{State of the Art}

Human Activity Recognition (HAR) is the foundation for a purposeful Ambient Assisted Living (AAL) approach. HAR approaches are typically classified by their usage of sensor types: intrusive or unobtrusive. Intrusive sensors are often more application-oriented than comfort-oriented. This gives researchers the opportunity to set up controlled experimental scenarios with a high information rate and quality. Every parameter can be adjusted, and all measured data can be deduced. However, these sensors are not ambient. Unobtrusive sensors are more practical for private households. For example a smart meter has the ability to detect active home appliances [13] or smart plugs placed in a socket to detect home appliances used. Small, mostly wireless sensors [14] or one device analyzing the power consumption [15] are also used to detect human activity. These sensors provide less detailed information than intrusive sensors, but they are accepted by the user.

A HAR system has to be trained before detection can be done. This can be classified into unsupervised and supervised. Supervised training uses a labeled training dataset, which represents normal activities in a household. For example, [14] deploys a very large number of extremely simple sensors to train naive Bayesian classifiers. These approaches perform well in HAR [16], but relying on labeled training data in private household scenarios has some drawbacks. For each household, a new training set is needed because every person has different habits. The monitored person has to $\log$ his/her activities to get a labeled training dataset. This procedure can be done with additional sensors, but this would not fit the assumption of unobtrusive sensors. Thus, it has to be done manually: either handwritten or with technical support like headsets and voice recognition [8] or with the help of a Personal Digital Assistant (PDA) [14]. This process is time-consuming and error-prone, because the person involved gets annoyed logging each and every activity or simply forgets to do so and tries to remember them later or just copies and pastes the activities. We already experienced those effects [11,12] in three different scenarios: (1) the elderly in private households; (2) young academics in private households and (3) mixed academics in offices. Logging the activities is also assumed intrusive and shall not be used in this paper's approach.

Early unsupervised learning approaches for HAR used an activity or inactivity level. In [17], a daily inactivity diagram is explained where a threshold function is learned to detect deviations. It cannot detect activities. In [18], a single index of daily activity is calculated to detect Activity of Daily Living (ADL) and long-term changes. It uses a fixed assignment of sensors to ADL. However, this assignment can vary from person to person. Our approach designs a HAR system where such an assignment will be individualized. 
Nowadays, HAR is based on event stream analysis, where a combination of different sensors refers to a specific ADL. This leads to the segmentation problem where the optimal length of a sensor stream has to be estimated to get the best HAR results. In [19], a dynamic windowing approach is used, but still has problems detecting tight occurrences of ADL within one data segment. The author in [20] solves this problem using a dynamic AES segmentation algorithm to detect also tight ADL. The dynamic segmentation solves the segmentation problem well, but it is based on a supervised training algorithm and is therefore not suitable for our approach.

Unsupervised training methods rely on datasets and/or ontologies, storing information to alleviate the need for manually-labeled training data. For example, a formal description language can describe human workflows [21]. It also can be used to detect executed activities by data mining. However, each workflow has to be defined manually and differs for each person. The project Unsupervised Semantic Mining Activity Recognition Technique (USMART) [22] uses ontologies, where spatial information and temporal information are analyzed to detect ADL. There are two ontologies: the sensor-room ontology and the sensor-activity ontology. Data stream segments are clustered into ADL. Unfortunately, no parallel ADL can be detected. The aim of our approach is to provide an unsupervised learning algorithm needing only a few a priori data to detect ADL and parallel ADL. To summarize our requirements, the HAR system must:

- use unobtrusive, inexpensive and few sensors,

- work with unsupervised training,

- work with individualized assignments of sensors to ADL,

- detect parallel HAR,

- not rely on complicated a priori information.

\section{Structure Detection in the Markov Model}

\subsection{Initial Setup and Training Data}

The HAR-system acts in three stages: initial phase, training phase and application phase. In the initial phase, a priori knowledge is gathered. In our case, it is a list of sensors used preliminarily assigned to possibly detectable ADL by each sensor. This assignment is an n:m assignment, which means that one sensor can lead to multiple ADL, like the fridge sensor can be used in the ADL prepare food, take a drink or store groceries. On the other hand, one ADL can be detected by multiple sensors, as well, like the ADL prepare food can include the usage of the fridge, the water tap, the oven and the cupboard with plates. This assignment is general knowledge, leviable with low effort and not final. It will be changed and adjusted for the monitored person in the training phase by learning a probability distribution function (pdf) in Section 6. This pdf tells how strong a sensor event points to one certain ADL. For example, Person A: the usage of the fridge points with a probability of 0.6 to the ADL "have a snack" and with 0.2 to "prepare food"; whereas Person B does use the fridge more often for "preparing food" with a probability of 0.5 , and "have a snack" only reaches a probability of 0.1 (or even zero). This probability distribution is learned without supervision in the training phase. During the training phase, all models of the HAR-system will be trained, and this will be used within the application phase. Both phases will be explained in the remaining sections of this publication.

Human activities have to be deduced from sensor data. For our approach, a sensor must deliver a start and end time of the sensor's activation. Therefore, each sensor and sensor type that can deliver such information is suitable for our HAR. For example, only one smart meter could be used for a whole apartment. The smart meter must be able to detect which appliance (device ID) was turned on and off with the corresponding time stamps [11]. Another example could be the usage of a motion detector. In this case, a motion sensor must be installed in each room or zone where an activity shall be detected. Therefore, our approach is independent from sensor types and only needs the most basic sensor information of a timestamp, a sensor ID and the sensor's state (on/off, active/inactive, open/close). The result is an ordered sensor event stream, which looks like the following: 
- 2017-02-25 11:33:41 ID 8 sem Fridge State on

- 2017-02-25 11:35:33 ID 8 sem Fridge State off

- 2017-02-25 11:36:43 ID 8 sem Fridge State on

- 2017-02-25 11:37:04 ID 7 sem Cupscupboard State on

- 2017-02-25 11:39:00 ID 7 sem Cupscupboard State off

- 2017-02-25 11:40:23 ID 8 sem Fridge State off

- 2017-02-25 11:41:00 ID 5 sem Hall-Toiletdoor State on

- 2017-02-25 11:41:24 ID 14 sem ToiletFlush State on

- 2017-02-25 11:42:55 ID 14 sem ToiletFlush State off

- 2017-02-25 11:43:14 ID 5 sem Hall-Toiletdoor State off

- 2017-02-25 11:44:28 ID 8 sem Fridge State on

- 2017-02-25 11:45:01 ID 8 sem Fridge State off

- 2017-02-25 11:46:58 ID 8 sem Fridge State on

- 2017-02-25 11:47:01 ID 8 sem Fridge State off

For example the event "2016-02-25 11:41:24 ID 14 ToiletFlush State on" tells that the toilet flush with its ID number 14 was turned on at 11:41:24 in 2017 on 25 February. The toilet flush obviously refers to the ADL toilet usage; whereas instead, it is not possible to assign the sensor event "2017-02-25 11:36:43 ID 8 sem Fridge State on" to one ADL only. The fridge can be assigned to many ADL, for example: eating, preparing breakfast/dinner/lunch, drinking, having a snack and storing groceries. The next sensor event might be helpful "2017-02-25 11:37:04 ID 7 sem Cupscupboard State on". Cups can be used in the ADL eating, preparing breakfast/dinner/lunch and drinking, but not in have a snack or storing groceries. Thus, a combination of events is needed to deduce the executed ADL.

\subsection{Human Activity Recognition (HAR)-Model}

A model representing sensor event (E) combinations is needed. We decided to take a Markov Model (MM) that is easy to design. Each MM-state $Z$ represents a set of currently "on" sensors $(\mathrm{Z} \subseteq \mathrm{E})$. A change of state occurs if a sensor's state changes. Each MM-state logs the entry and exit times (start time and end time). If no sensor is "on", the MM returns to an idle-state. This idle-state always exists. It cannot be learned or eliminated during the training phase. This state also collects all ADL that are not measureable by sensors. In other words, it represents the inactivity of a person. This idle-state is the center of the MM and a starting point for all of the following analysis methods.

An example part of the MM can be seen in Figure 1. It includes the states (green rectangle) derived from the data stream example above and an additional part (blue rounded mark) to show one MM-structure. Such a structure is a temporal correlation of sensor events, which is separated from the rest of the MM and thus represents a human habit. Most structures that can be found in such an $\mathrm{MM}$ are formed as stars: one MM-state is the start and end point for most connected paths. A more detailed description of all possible structures can be found in a former publication [23]. The structures are detected during the training phase. The first step of training is to read the off-line sensor data. Given the MM-state definition and the fact that reading the measurements equals an ordered event stream, structures can be found while importing the offline training data. For example, all paths (circles) starting at MM-State \#1 and leading back to MM-State \#1 are considered to be one structure. More detailed information about the structures and their detection can be found in a former publication [23]. The training data are countable finite. Only a representative amount of sensor combinations is contained. During the ADL-recognition phase, not yet learned combinations can occur, but still must be recognized. Therefore, the training result must be flexible (uncertain) enough to recognize similarities. This is achieved by using a probability distribution function of sensors influencing the ADL detection, which is explained in the next sections. 


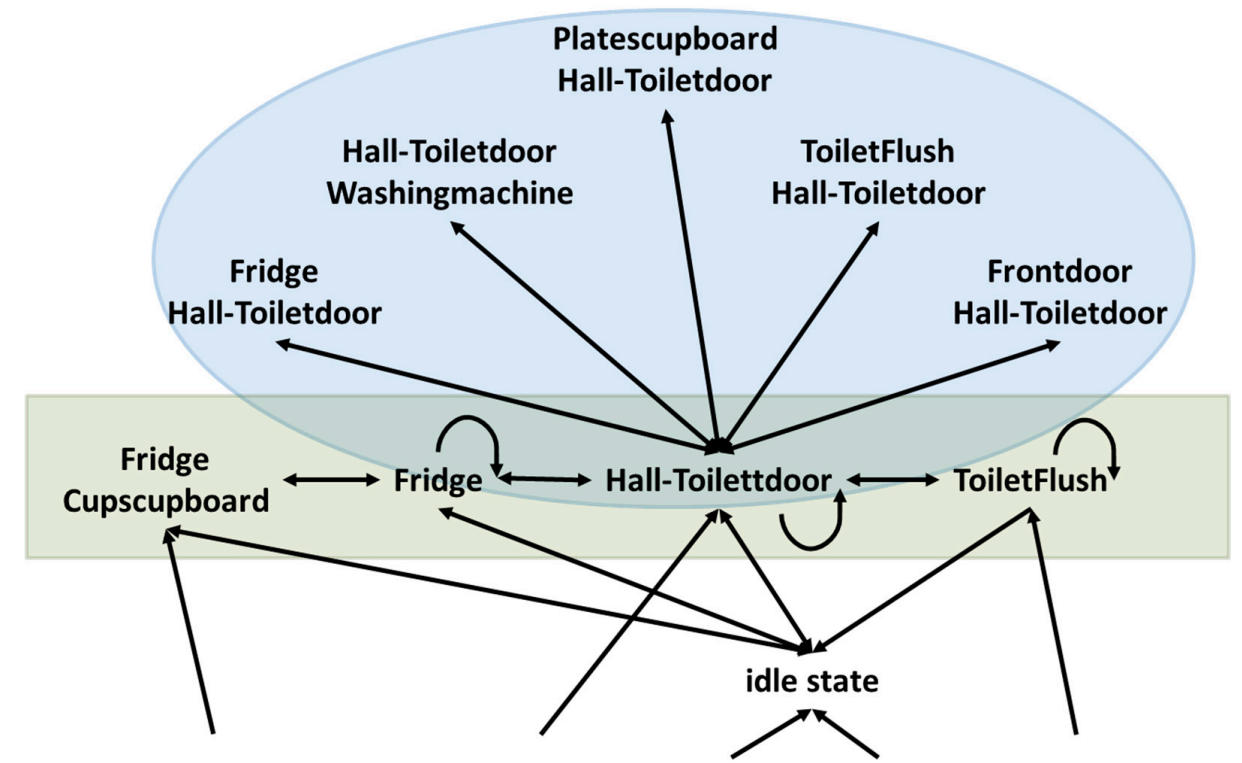

Figure 1. The part of the learned Markov Model (MM) showing an example (green rectangle) of two nested Activity of Daily Living (ADL): drinking and use toilet. A typical MM-structure: a star is seen in the blue rounded area.

\section{Markov Model (MM)-Structure Significance Estimation}

The MM-structures represent individual learned human behavior patterns. To characterize these patterns, they are rated in comparison to the corresponding ADL-MM. An ADL-MM is an MM learned only by the sensor data related to one ADL. As described in Section 3, sensors are assigned to a couple of ADL. Therefore, only the assigned sensors are considered while learning an ADL-MM. The structural characteristics are rated by a significance value $0 \leq G_{S} \leq 1$ for each ADL $a_{i}$. $G_{S}$ is computed by:

$$
G_{S}=0.6 \frac{N_{m T}+N_{m Z}}{\max \left(N_{T_{1}}, N_{T_{2}}\right)+\max \left(N_{Z_{1}}, N_{Z_{2}}\right)}+0.2 \frac{N_{S A}}{N_{T A}}+0.2 \frac{\sum_{i=0}^{N} o c c_{i}}{\sum_{i=0}^{N} o c c A D L_{i}} .
$$

The equation combines the comparison of structural sub-criteria and statistical sub-criteria as summarized in Table 1. The first sub-criterion of $G_{S}$ is the similarity of two structures. It is calculated by the ratio of matching states and matching transitions in relation to the sum of the maximum of states and transitions of the ADL-MM or structure. The matching of identical states is simple, as they are distinctly defined by the set of active devices. The transitions are distinctly identified by the tuple of source states and target states. As states and transitions are the base of an MM, this sub-criterion has a major influence and is weighted with $60 \%$ within the $G_{S}$. The weights used in Equation (1) are retrieved by experience from former experiments. A full sensitivity study still has to be done, but the results are already good. The weights are final. In future work, it may be considered to learn these parameters or make them adjustable suiting the current situation. However, for our purpose, they stay static. We chose the weight according to the relevance of the sub-criterion. Therefore, the structural characteristics have a majority influence and the accessibility and the frequency of occurrence a minor influence. The degree of accessibility rates the variation of transitions to leave or enter a structure. It ranges from $0-1$, while a higher value represents additional transitions to enter or leave. The more often this structure is entered from different states, the higher the probability that this human habit can occur. The third sub-criterion is a ratio calculation of the occupancy of the structure and the occurrence of the ADL-MM. The structure significance estimation $G_{S}$ represents the relevance of the structure and will be used in the following sections of this paper as a weight. 
Table 1. Sub-criteria of the calculation for structural significance. Each sub-criterion is described by its equation part, and the parameters are named below.

\begin{tabular}{lc}
\hline Sub-Criterion & Equation Part \\
\hline Similarity of the structure with the MM & $\frac{N_{m T}+N_{m Z}}{\max \left(N_{T_{1}}, N_{T_{2}}\right)+\max \left(N_{Z_{1}}, N_{Z_{2}}\right)}$ \\
\hline Degree of accessibility & $\frac{N_{S A}}{N_{T A}}$ \\
\hline Strength of structural components & $\frac{\sum_{i=0}^{N} o c c_{i}}{\sum_{i=0}^{N} o c c A D L_{i}}$ \\
\hline$N_{m Z} \quad$ number of matching states \\
$N_{m T} \quad$ number of matching transitions \\
$N_{S A} \quad$ number of entry and exit transitions \\
$N_{T A} \quad$ number of transitions without match: $N_{T}-N_{m T}$ \\
$N_{T} \quad$ number of transitions \\
$N_{Z} \quad$ number of states \\
$o c c_{i} \quad$ occurrence of a structure part $i$ \\
$o c c A D L_{i} \quad$ occurrence of an ADL part $i$ \\
$N \quad$ maximum of states numbers in all parts $i$ \\
\hline
\end{tabular}

\section{Human Forgetting Curve Impulse}

\subsection{Human Forgetting Curve}

The human habit significance derived from the MM described in Section 4 has two drawbacks. First, a human has a longer memory capacity than the MM, the memory of which only reaches up to one state in the past. Second, a human can execute more than one ADL in parallel. Especially, the ADL media are often executed in the background, or the usage of the toilet is very often in the background, as the example in Figure 1 shows. Thus, an additional method needs to be added to the MM-approach. The human forgetting curve [24] in Figure 2 was developed by psychologists to represent the memorized percentage over time.

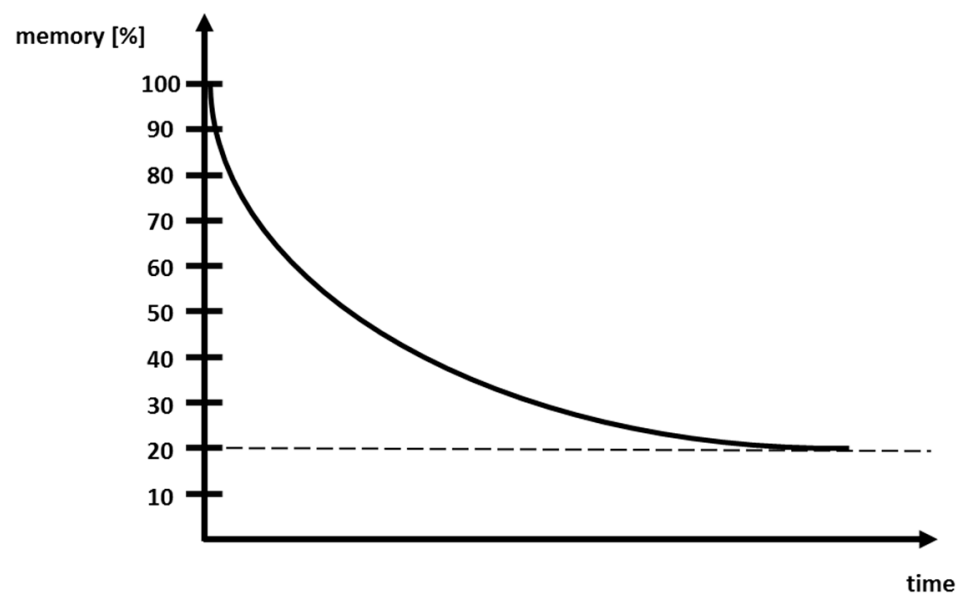

Figure 2. Human forgetting curve. This shows the human memory capability and that the human awareness decreases over time following an exponential function.

Human memory diminishes over time. Furthermore, even healthy young humans forget. Experiments evaluated that a human forgets newly learned random data: after $20 \mathrm{~min}$, the human

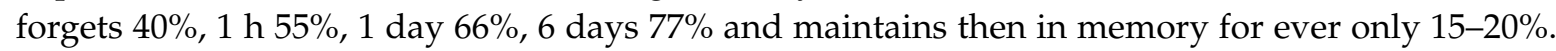
These data vary by age, type of learning, the number of repetitions and the type of data that are learned. For example, data that have a certain logical relation remain longer in memory than randomly-generated numbers. The more often the data are repeated, the longer they stay in memory. 
The forgetting curve decreases as an e-function that has to be parameterized for each situation. This is done in the next section.

\subsection{Impulse Approach}

The human forgetting curve impulse (hfci) analyzes the temporal correlation of events. If two events occur in quick succession and are assigned to the same ADL, the ADL's significance is increased; while longer time intervals between events have a reduced impact. This is modeled by an exponential decline function for each ADL. If a sensor is activated, the corresponding ADL are stimulated according to their relevance weight $G_{a}$. The calculation of this weight is explained later in this section. The stimulus impulse may not drop below $20 \%$ as long as the sensor is active. The stimulus declines after the sensor is deactivated. The hfci $I_{a_{i}}(t)$ of each ADL $a_{\mathrm{i}}$ is calculated by:

$$
I_{a_{i}}(t)=\sum_{e \in \mathrm{E}}\left\{\begin{array}{l}
G_{a}\left(e, a_{i}\right)\left(0.2+0.8 e^{-d\left(t-t_{o n}\right)}\right), \text { active } \\
\left.G_{a}\left(e, a_{i}\right) b_{\mathrm{on}} \times e^{-d\left(t-t_{o f f}\right)}\right), \text { inactive }
\end{array}\right\}
$$

with the time $t$ and the relevance weight $G_{a}\left(e, a_{i}\right)$ of each home appliance $e$. As described in Section 5.1, the human forgetting curve by Ebbinghaus is designed to keep at least $20 \%$ of learned content in memory. Thus, the hfci for active sensors starts at a value of 0.2 , and the exponential decline is decreased to $80 \%$. This means that without $G_{a}$, the maximum outcome is one $(100 \%)$. The factor $G_{a}$ is able to increase or decrease the hfci's behavior. The factor $d$ describes how fast the curve decreases over time. It can be derived by the descriptions in Section 5.1, as well. For example, after $20 \min \left(t-t_{o n}=1200 \mathrm{~s}\right)$, a human forgets $40 \%$. This means that $60 \%$ will be remembered and $I_{a}$ is 0.6 . The factor $G_{a}$ can be neglected for these adjustments because $G_{a}$ shall not have any influence on the way of forgetting. It only shall influence the maximum height.

Figure 3 shows an example of the hfci. Each function curve represents one ADL. It is the corresponding hfci from the MM shown in Figure 1.

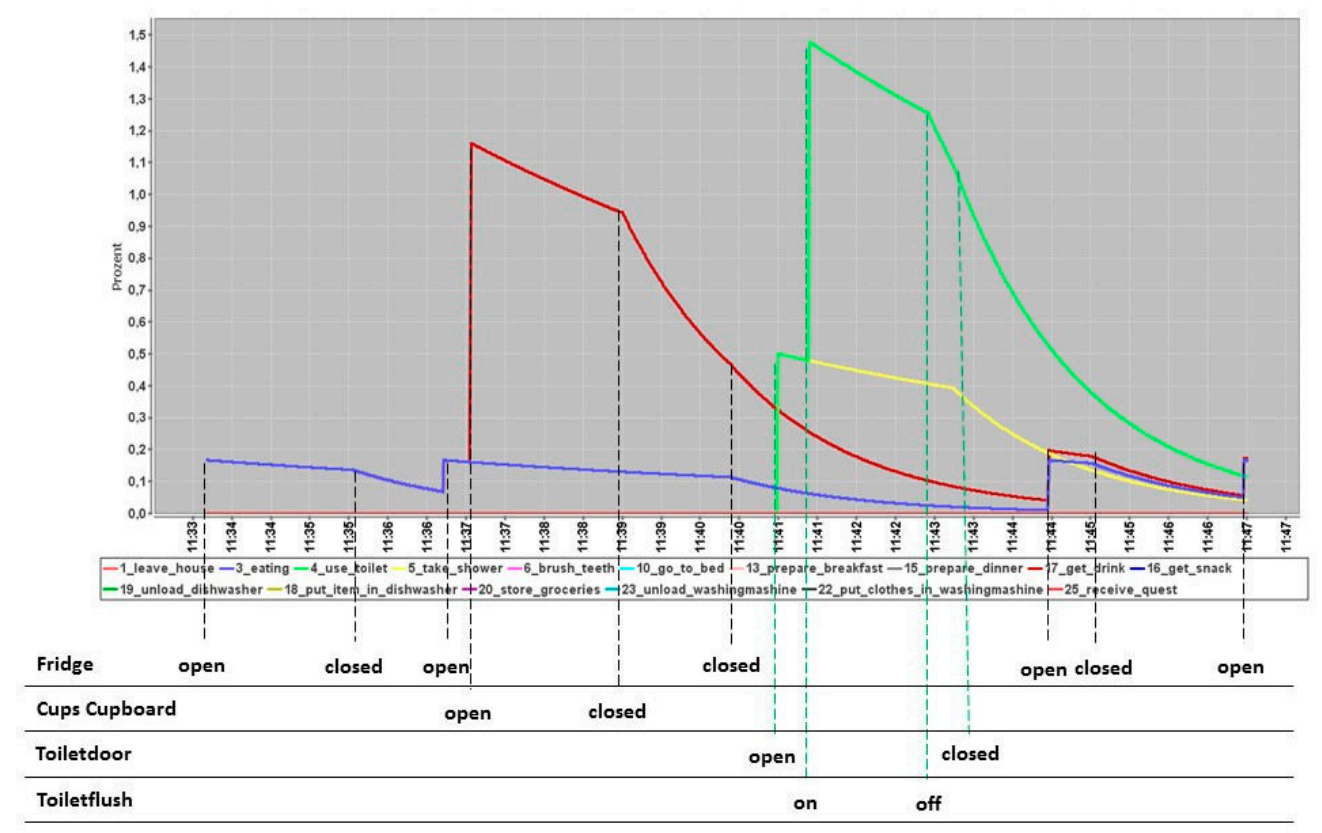

Figure 3. The human forgetting curve impulse (hfci) with the equal distribution of sensor-ADLassignment (sensor probability distribution function (spdf)). Each colored function curve represents the impulse curve hfci for one ADL. The table underneath the curve diagram shows the corresponding sensor events. 
The upper part of the picture shows the hfci-diagram, and the lower part shows the corresponding sensor events. Between 11:33 a.m. and 11:47 a.m., some ADL overlap. The blue curve (ADL: eating) and the dark red curve (ADL: take drink) seem to compete to be the executed ADL. Additionally, the light green curve (ADL: use toilet) and yellow curve (ADL: take shower) also seem to compete. Additionally, the winning ADL of both competitions seem to be executed in parallel. The remaining ADL are too minor to be considered. Therefore, four ADL, eating, take drink, take shower and use toilet, are the dominant ADL, and the algorithm has to choose the executed ADL among them. To estimate which ADL is the dominant ADL, the area under each hfci is calculated. However, the challenge is to determine a start and end point for these calculations. These calculations are part of the training phase. We face this segmentation problem by using the learned MM-structures from Section 3. These structures represent human habits, for which we still do not know to which ADL they belong. By calculating the area under each ADL-hfci, a ranking from the highest to the lowest value can be generated. The highest area value is obviously the most dominant ADL. For the example in Figure 3, it would be the dark red curve (ADL: take drink) and the light green curve (ADL: use toilet).

The influence of the human memory capacity of the hfci can be seen at 11:44 a.m. The cupboard was already closed at 11:39 a.m., but the memory kept this event in mind by a slow decrease of the function curve. The next event assigned to the ADL take drink (red curve) is added to a higher current value than is the case with the ADL eating (blue curve). Thus, the more dominant ADL between these two is still the ADL take drink. Furthermore, a new sensor event Fridge "on" occurred at 11:44 a.m., which is equally distributed to both ADL eating and take drink. This memory capability can also be misinterpreted. At 11:44 a.m., the ADL use toilet and take shower are still higher than the curves assigned to the current event red and blue (ADL take drink and eating); also, all sensors for the ADL use toilet and take shower are already deactivated. Therefore, the detection of the current executed ADL can be delayed due to the memory capability of the hfci. This problem will be addressed in the next Section 6.

Until now, the hfci obviously depends on the number of activated sensors and their elapsed time. As is known from Section 3, the sensors are assigned to ADL a priori. From this assignment, the parameter $G_{a}$ is equally distributed. For example, the sensor Fridge is assigned to six different ADL eating, preparing breakfast/dinner/lunch, drinking, have a snack and storing groceries. Each $G_{a}$ is 0.167 . If the Fridge sensor turns "on", each assigned ADL has the same probability of 0.167 to be the detected ADL. However, this assignment is static and can differ from person to person. A distribution function of how much a sensor refers to an assigned ADL is needed. This is explained in the next section.

\section{Sensor Probability Distribution Function}

Each sensor was assigned to the logical corresponding ADL during the initial phase. For the fridge example in Section 5.2, an equal probability distribution would refer to each ADL with a probability of $G_{a}=0.167$. This is too static and needs to be individualized, because the $G_{a}$ distribution is obviously different for each human. Without the individualization, the whole ADL-detection would be like a majority vote where the ADL wins for which the most sensor events were assigned. To avoid this, the sensor probability distribution function (spdf) is trained, which states how strong a sensor event refers to one ADL. For example: there are three sensor events assigned to ADL "preparing food" and to the ADL "take a drink". The sensor events may be: fridge opened, cupboard opened, water tap used. The spdf learned may result in the spdf assignment between sensor event and ADL as follows:

- fridge opened: ADL "take a drink" = 0.3 and ADL "prepare food" $=0.6$

- cupboard opened: ADL "take a drink" $=0.6$ and ADL "prepare food" $=0.2$

- water tap used: ADL "take a drink" $=0.4$ and ADL "prepare food" $=0.3$ 
This assignment would sum up for the ADL "take a drink" $(0.3+0.6+0.4)$ to a total of 1.3 and for the ADL "prepare food" $(0.6+0.2+0.3)$ to a total of 1.1. With such a calculation, it is also possible that the minority of sensor events can win. It all depends on the learned spdf.

To calculate the spdf, the information from previous sections is needed:

- $\quad$ structurally important parts of the MM representing strong human habits (Section 3),

- $\quad$ structurally significant $G_{S}$ defining the influence of a human habit (Section 4),

- $\quad$ sorted list of the area underneath each hfci; representing an ADL ranking (Section 5).

First, the hfci for each structure is calculated. Then, the area underneath is calculated, which leads to a list of ranked ADL (LdomADL) where the first element is the most dominant, which is the most probable executed ADL. This list of ranked ADL also includes parallel executed ADL. The detection of parallel ADL is explained later in Section 7.

Now, we are looking at each single sensor involved in the structure and calculate the spdf by doing the following. Each sensor has a list of assigned ADL (LS). For each ADL $a \in \operatorname{LdomADL}$ and $a \in L S$, the spdf will be increased by the summand:

$$
d=\frac{G_{S}(a)}{\left|L_{\text {dom } A D L}\right|}
$$

where $|L d o m A D L|$ represents the number of dominant ADL. Therefore, the value representing the relevance of a human habit is used to increase the sensor's probability to refer to a dominant ADL. The spdf of non-dominant ADL has to be decreased by:

$$
d=\frac{G_{S}(a)}{\left|L_{A D L}\right|-\left|L_{\text {dom } A D L}\right|}
$$

where $|L A D L|$ represents the number of all assigned ADL to the sensor. See Figure 4 for a visualization of this algorithm.

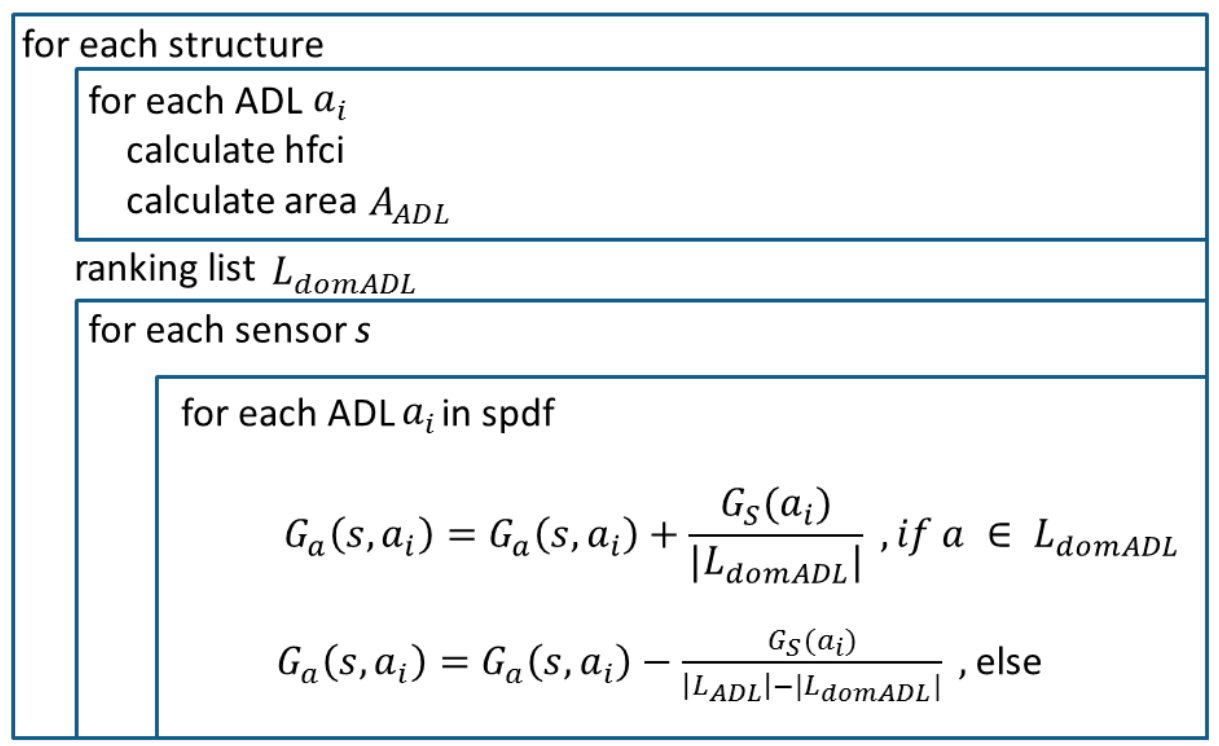

Figure 4. Algorithm for the spdf calculation. It shows one iteration, which is repeated until no changes occur anymore.

Figure 5 shows the resulting hfci-diagram after training the spdf for this one human pattern of the example given in Section 3. Figure 3 shows the same diagram, but without the individualized spdf. Figure 5 has a more clear detection of ADL where only two ADL, take drink (red curve) and use toilet 
(light green curve), remain to be dominant ADL. It is now possible to deduce the executed ADL more precisely. Training data are much larger than this small example and have a lot more sequences. Each sequence influences the spdf anew until a stable spdf is reached.

With the individualization of the spdf, the training phase is completed. Each sensor installed in the private household is now assigned to a list of ADL with an individualized probability expressing the bonding of a sensor to an ADL. The next section explains how the spdf is used to detect ADL.

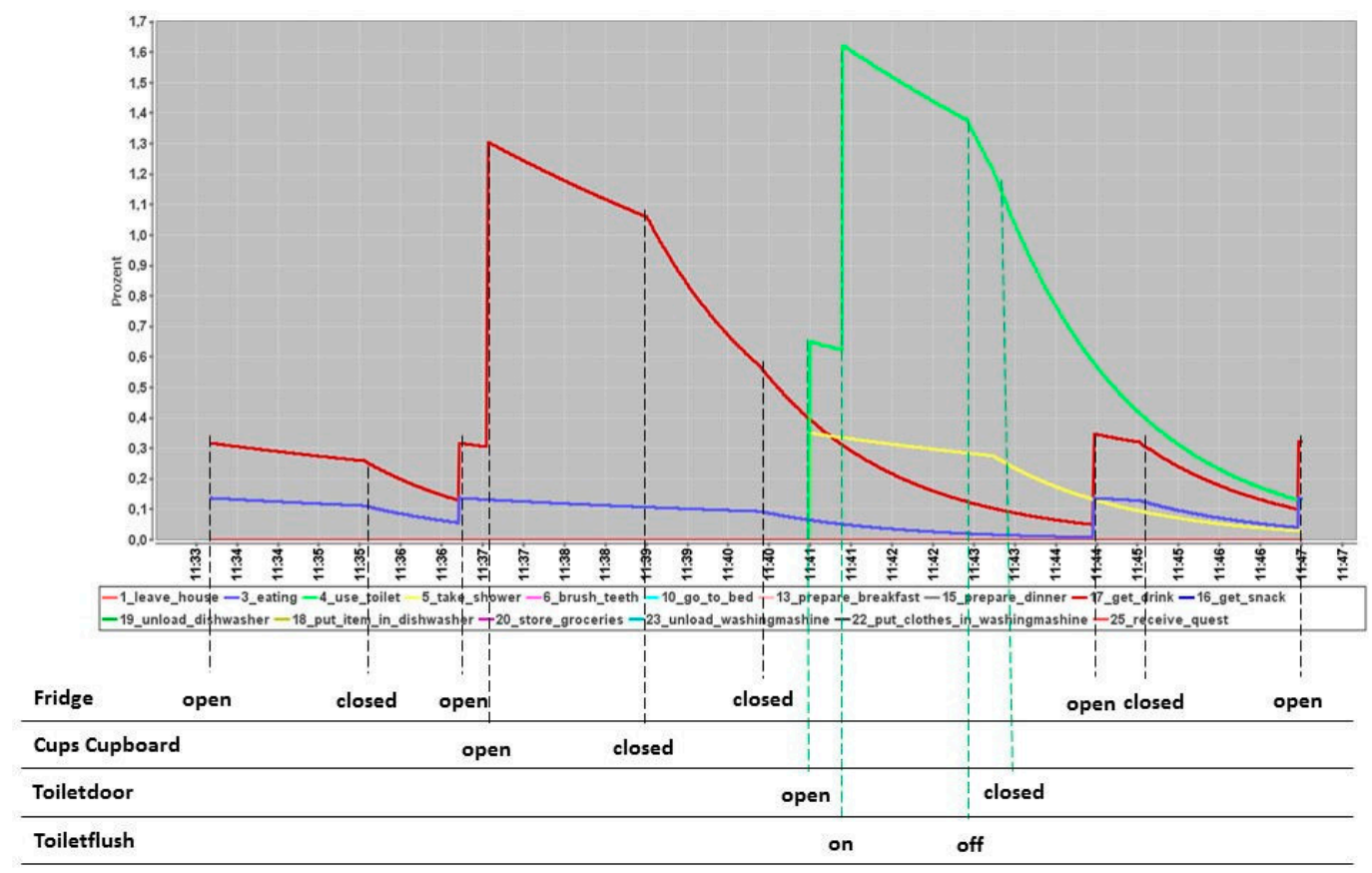

Figure 5. The hcfi after learning the sensor-ADL-assignment (spdf) for 16 ADL. Each colored function curve represents the impulse curve hfci for one ADL. The table underneath the curve diagram shows the corresponding sensor events.

\section{Activity of Daily Living (ADL) Detection}

The main aim of this approach is to detect ADL in single private households. In the previous Section 6, a probability distribution function representing the influence of a sensor (spdf) on the ADL detection was introduced. This learned spdf is needed to calculate a probability distribution of detected ADL (apdf). Due to the complex calculation for training, the ADL detection can be short and efficient and is abstractly shown in Figure 6.

Every time a new event occurs, the following steps are executed. First, the current hfci values for each ADL are calculated. Only the current value is needed to analyze present and historical data influence, because it includes the human memory capability. Therefore, the problem of finding the best sequence length is neglected. These current hfci values lead to an ADL ranking where the highest value represents the most dominant and most probable executed ADL. This ranking will be transformed into the apdf. It easily can be transformed into a percentage distribution, which is far easier for humans to compare and understand. If the apdf is equal for some ADL, they are assumed to be executed in parallel.

Second, for each ADL, the set of sensors "on" is calculated. If sets of sensors are disjoint, these ADL are executed in parallel. If there is an ADL where the set of sensors is empty, but the hfci is positive, this ADL was the recent executed ADL. This one is still in the algorithm's memory and can become an interrupted (nested) ADL if a sensor event assigned to this memorized ADL occurs next. Following these rules, a probability distribution is calculated including parallel and nested ADL by dividing the hfci-value by the sum of all hfci-values. Parallel ADL obtains the same probability as the 
ADL where parallelism was detected. If two ADL have a very similar probability, then two cases are possible: first, these ADL could be interpreted as parallel or, second, it is not possible for the algorithm to decide more precisely which of the two ADL is the most dominant, and additional information may be needed.

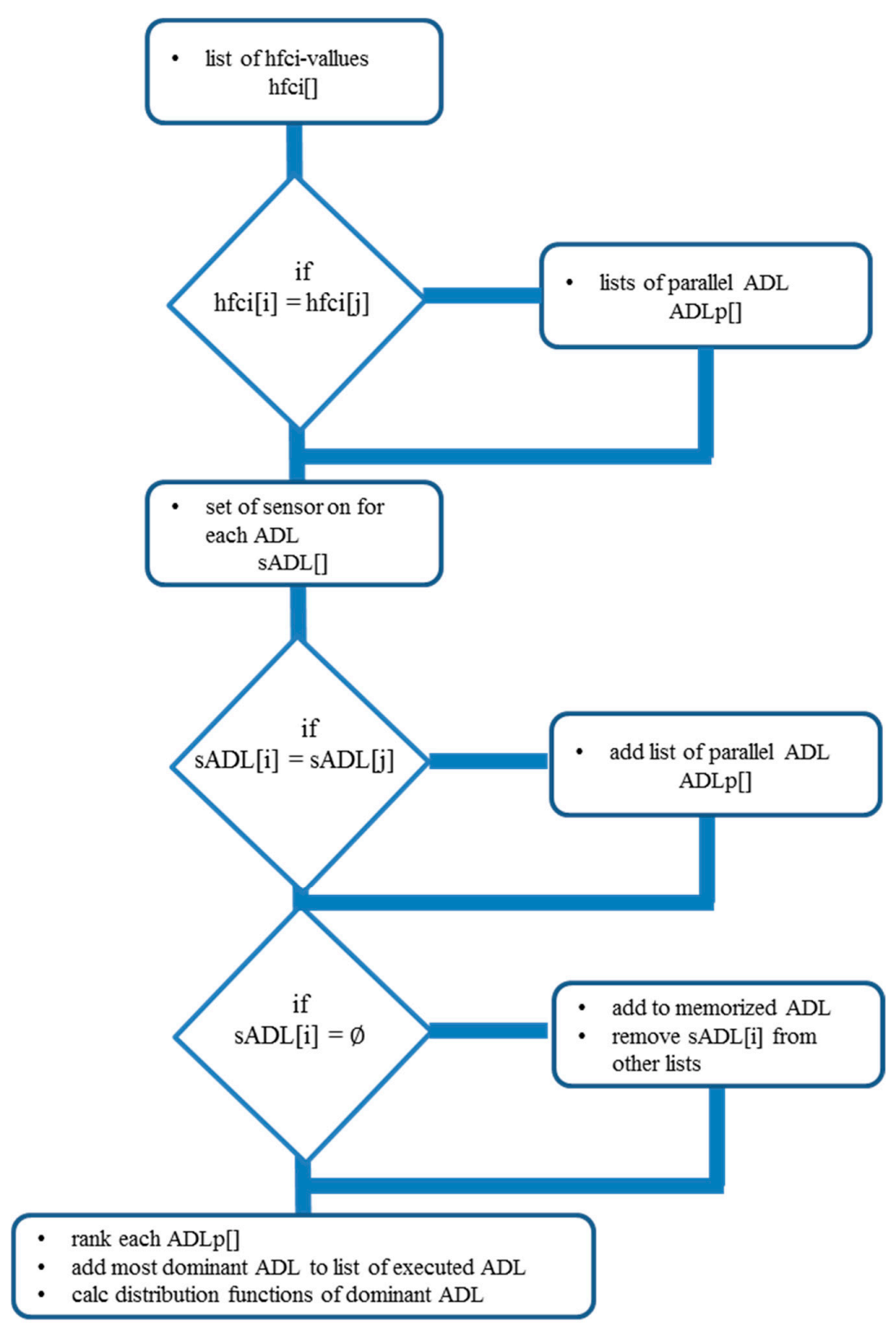

Figure 6. Abstract algorithm for the ADL detection. This chart shows the steps for the detection of parallel ADL.

\section{Evaluation and Discussion}

For the evaluation, a dataset with labeled ADL is needed. Our first attempt to get such a labeled dataset was to equip our own apartments and offices at the University of Technology Dresden with smart meters and ask the monitored persons to manually log their activities [11]. These handwritten logs were so time-consuming that the volunteers forgot logging or tried to remember it later or just copied the activities from a previous day. Therefore, we decided to use a well-labeled and publicly available dataset: the benchmark dataset from Kasteren [8]. This dataset includes three different datasets with totally different houses, persons, sensors and ADL. Each dataset includes two lists: one list of bias sensor events with the start and end times and a second list of ADL with the start and 
end times. The characteristics of the chosen datasets are shown in Table 2. Houses B and C are more complicated for HAR-detection, as they have over 300,000 events each and more sensors and activities to detect.

Table 2. Characteristics of the publicly available dataset that was used to evaluate the new approach of ADL detection. Three different datasets are used: Houses A, B and C.

\begin{tabular}{cccc}
\hline Item & House A & House B & House C \\
\hline Age & 26 & 28 & 57 \\
Gender & Male & Male & Male \\
Setting & Apartment & Apartment & House \\
Rooms & 3 (+2 Bathrooms, +1 Kitchen) & $2(+1$ Bathroom, +1 Kitchen $)$ & $6(+2$ Bathrooms, +1 Kitchen $)$ \\
Duration & 25 days & 14 days & 19 days \\
Sensors & 14 & 23 & 21 \\
Activities & 16 & 13 & 16 \\
\hline
\end{tabular}

Kasteren uses various kinds of sensors:

- $\quad$ reed switches to measure whether doors and cupboards are open or closed

- pressure mats to measure sitting on a couch or lying in bed

- mercury contacts to detect the movement of objects (e.g., drawers)

- $\quad$ passive infrared (PIR) to detect motion in specific areas

- float sensors to measure the toilet being flushed.

The benchmark does include the following models: naive Bayes, hidden Markov model, hidden semi-Markov model and Conditional Random Fields (CRF). All models need a supervised training approach. Their results show that an increase in model complexity generally results in an increase of performance [8]. The CRF produces the best results and will be taken for comparison. The HAR-system is trained with the whole dataset except one day. This one day is used for evaluation, as was done by the benchmark. Figures 7-9 show a graphical representation of the confusion matrices for the three houses. The calculated precision, recall, F-measurement and accuracy are shown in Tables 3-5 for each house separately, where the results of the new approach are also compared to the benchmark.

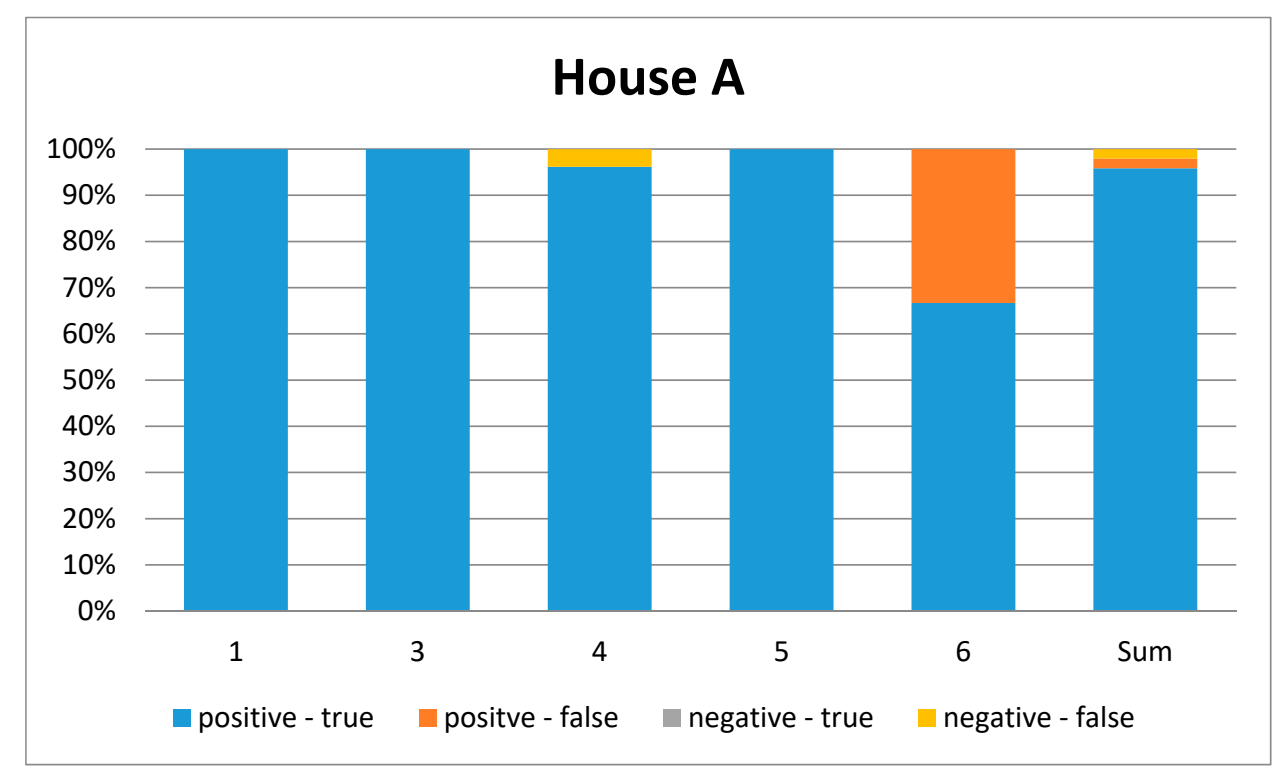

Figure 7. Graphical representation of the confusion matrix for House A. Each bar represents one ADL. ( 1 = leave house, 3 = eating, $4=$ toilet usage, $5=$ shower, $6=$ brush teeth). The last bar represents the overall ADL. 


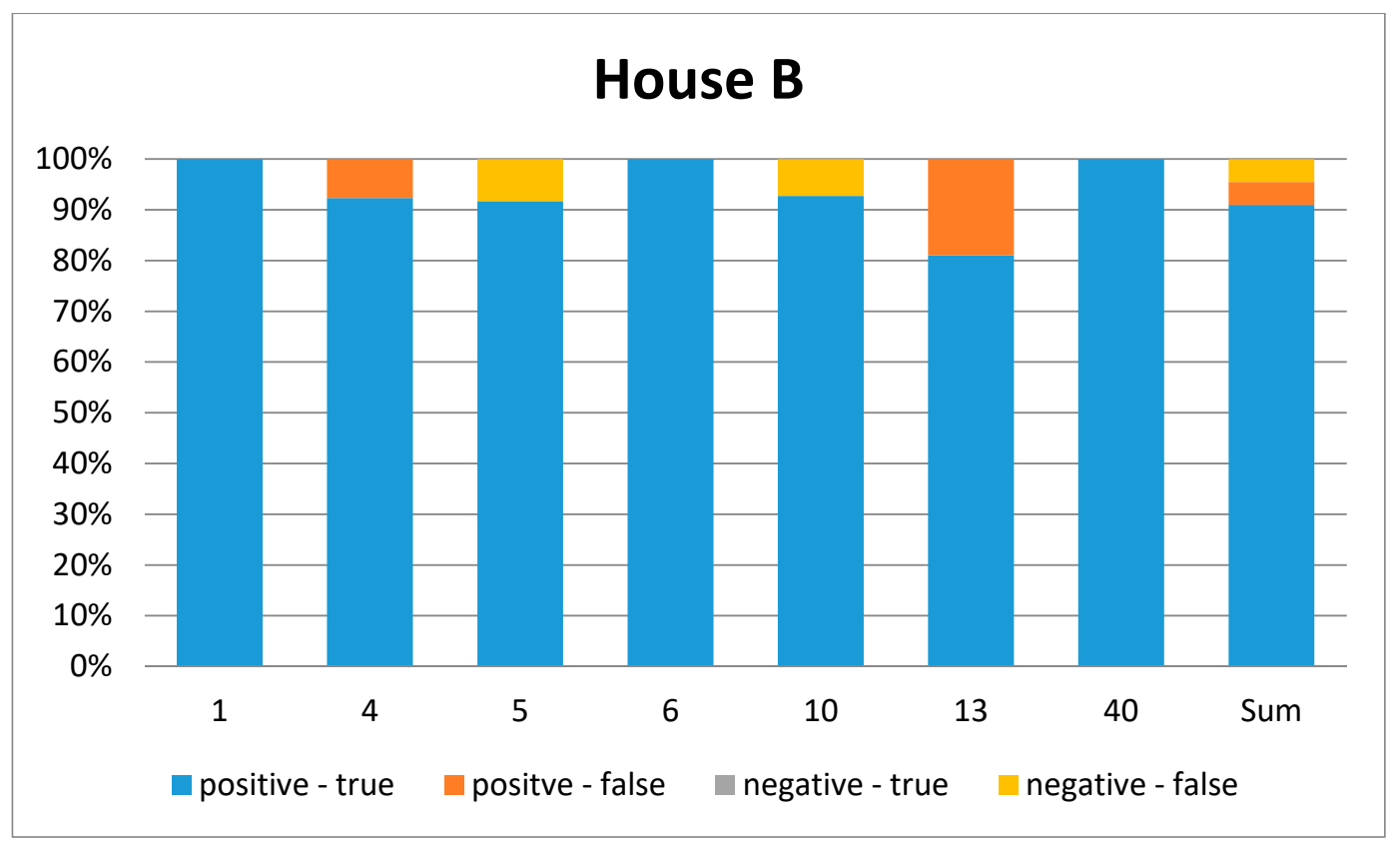

Figure 8. Graphical representation of the confusion matrix for House B. Each bar represents one ADL. ( $1=$ leave house, $4=$ toilet usage, $5=$ shower, $6=$ brush teeth, $10=$ sleep, $13=$ prepare breakfast, $40=$ play piano). The last bar represents the overall ADL.

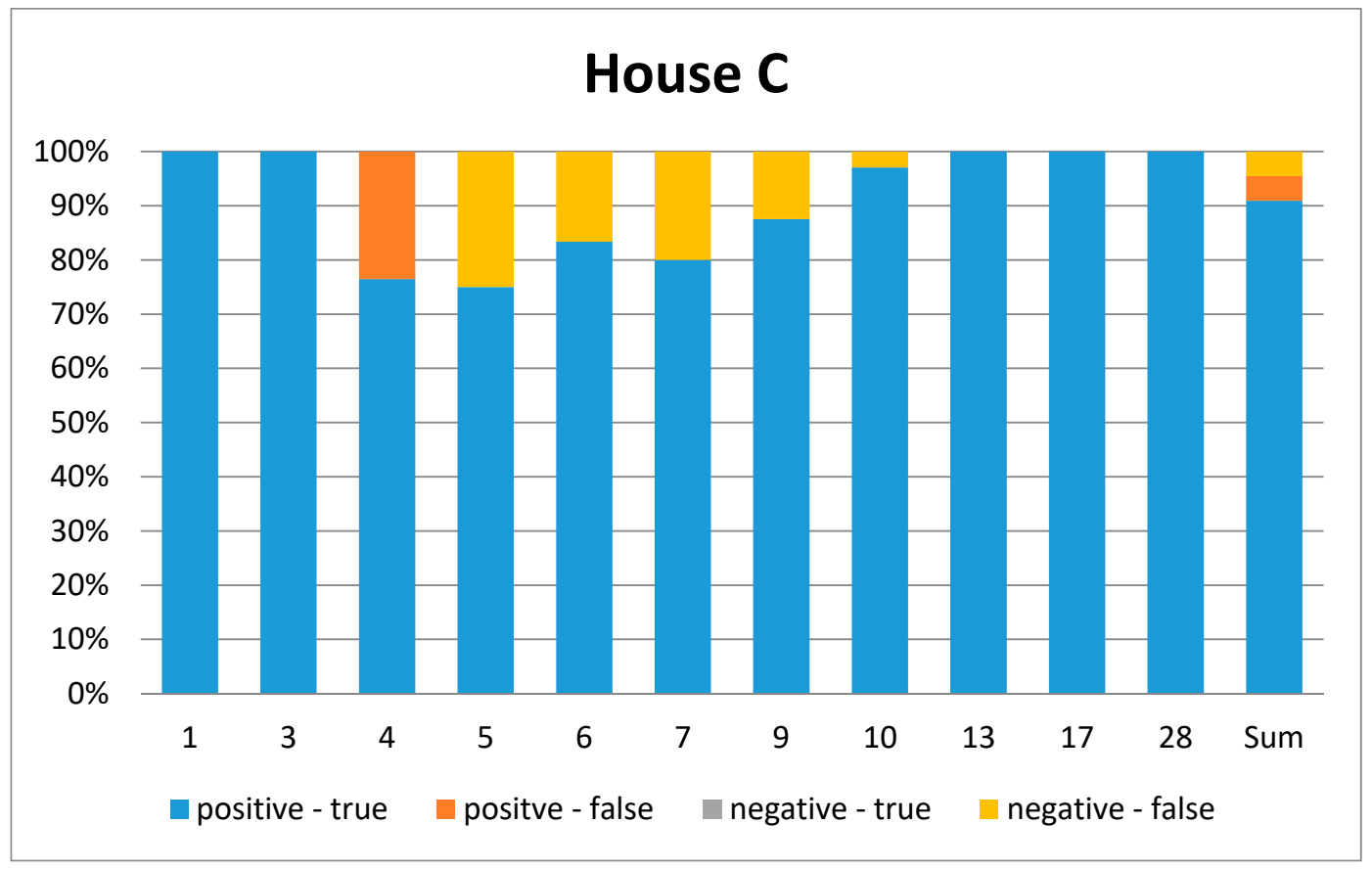

Figure 9. Graphical representation of the confusion matrix for House C. Each bar represents one ADL. ( $1=$ leave house, $3=$ eating, $4=$ toilet, $5=$ shower, $6=$ brush teeth, $7=$ toilet usage upstairs, $9=$ shaving, $10=$ sleep, 13 = prepare breakfast, 17 = drink, $28=$ relax). The last bar represents the overall ADL. 
Table 3. Precision, recall, F-measurement and accuracy in \% of House A in comparison with the benchmark (CRF).

\begin{tabular}{ccc}
\hline Criterion & CRF & Approach \\
\hline Precision & 73.5 & 99.2 \\
Recall & 68.0 & 93.3 \\
F-Measure & 70.4 & 95.6 \\
Accuracy & 91.4 & 97.9 \\
\hline
\end{tabular}

Table 4. Precision, recall, F-measurement and accuracy in \% of House B in comparison with the benchmark (CRF).

\begin{tabular}{ccc}
\hline Criterion & CRF & Approach \\
\hline Precision & 48.3 & 98.5 \\
Recall & 51.5 & 97.0 \\
F-Measure & 49.7 & 97.7 \\
Accuracy & 92.9 & 97.5 \\
\hline
\end{tabular}

Table 5. Precision, recall, F-measurement and accuracy in \% of House $\mathrm{C}$ in comparison with the benchmark (CRF).

\begin{tabular}{ccc}
\hline Criterion & CRF & Approach \\
\hline Precision & 36.7 & 93.3 \\
Recall & 39.6 & 98.5 \\
F-Measure & 38.0 & 95.5 \\
Accuracy & 82.2 & 97.0 \\
\hline
\end{tabular}

Furthermore, the difficulty increases from House A to C, but the detection accuracy stays almost the same. The CRF benchmark considers different feature representations analyzing the raw states, state changes or the last sequence state. We choose the state change representation because our approach also analyzes the state change. All CRF results are based on sequences. This means precision, recall, F-measurement and accuracy are calculated for each input sequence, and from these values, the average is taken. In our approach, we do not have input sequences, as it was our aim to neglect this problem of choosing the best sequence length. Therefore, we tried to adapt this by evaluating for each ADL which is the most suitable approach, because the benchmark detects one ADL in each segment.

Major issues occurred in the dataset of house B where motion detection sensors were used that randomly sent messages even though no person was there. We assume this to be an automated signal like a heartbeat. It is recommended to filter such signals before using the dataset so learn or evaluate. This can be done if the time of the heartbeat is known. Another issue is ADL that cannot be detected by any used sensor, like the ADL dressing. Even though a motion sensor close to the wardrobe is installed, no sensor has sent any message while the person annotated the ADL dressing. Therefore, we assume that the clothes were lying somewhere else in the bedroom or bathroom (after ADL taking a shower). It was also hard to choose a well-annotated day for the evaluation. The first criterion was to choose a full day of $24 \mathrm{~h}$ of annotations. The second criterion was to have a complete covered annotation. The first criterion was easy to fulfill. The second criterion was a challenge. The annotation quality differs from day to day and even from hour to hour. For example, the ADL take a shower and toilet usage had to be annotated. Sometimes, both were always annotated even when they occurred close to each other, and sometimes, the toilet usage in between showering was not annotated. This would also be a challenge for supervised algorithms. Another challenge is ADL that use exactly the same sensors always; like brushing teeth and shaving the face. Both are in the bathroom; both use water; and both tools were in the same cupboard. For the new HAR-algorithm, both ADL obtain the same probability for which the benchmark had to decide between them. This may be a reason why the new 
proposed HAR-system performs better. Another reason may be that Houses B and C also include parallel annotated ADL like using the toilet while playing piano. All parallel or nested ADL were successfully detected by the new HAR-approach. The benchmark is not able to detect such parallel or nested occurrences. The benchmark uses time slices where only one ADL for each time slice can be detected. Thus, some ADL remain undetectable by the benchmark, but are detectable with the new approach.

Overall, the new HAR-approach performs well with all three datasets where also the complexity factor increases from House A to B. An average accuracy of $97 \%$ was derived in all three datasets. Thus, the new approach outperformed the benchmark, where the best benchmark's result was $92 \%$.

\section{Recap and Outlook}

The introduced approach is a combination of a Markov Model (MM) and a psychological human memory model. The MM-approach is able to model sensor events in parallel by combining them in states. Therefore, it does not require the sequential and non-interleaving assumption of most other approaches. On the other hand, it can calculate a weight for a human habit $G_{S}$ for a sequence of MM-states, but ADL executed in parallel cannot be detected. Therefore, we introduced a human memory model that is not based on a state model, but analyzes the temporal correlations of the sensor events. Each ADL is modeled separately such that parallelism and temporary effects of nested ADL are easy to detect. The major advantage is that this combination does not need a labeled training set. It can be trained unsupervised. It only needs a preliminary defined list of sensor events assigned to ADL where each association can be an $n: m$ associated. This list will be individualized during training. The individualization is modeled by a probability distribution function, and thus, the result for the ADL detection is also a probability distribution function (apdf). The approach is able to calculate the most probable ADL executed and is also able to detect if another ADL is executed in parallel. Using more specific sensors, we can neglect this uncertainty, but this does not meet our requirements, which is to use inexpensive ambient sensors.

The approach can still be improved by adding a time of day-specific model. For example, the dataset from Kasteren [8] differs between the ADL eating, preparing breakfast, preparing dinner, get snack and get drink. Most sensor events are the same for all five ADL. By analyzing the time of day, the separation between these ADL could be more accurate.

\section{Conclusions}

The introduced approach is done via unsupervised training. Only a few general a priori pieces of information are needed, like which sensors are used and which ADL may detect them. This preliminary assignment will be adjusted to fit the person monitored by the HAR-system. A Markov model is built based on sensor events, and specific state patterns are detected. These patterns represent human habits and are rated by a significance factor. The Markov model lacks the capability of human memory. Therefore, the Markov model is combined with a psychological human memory model. The memory model describes the forgetting over time and overcomes the general assumption that ADL have to be executed subsequently. Instead, it allows the detection of parallel and nested ADL by calculating a probability distribution function for executed ADL (apdf). This apdf distinguishes between the most dominant executed ADL and the most dominant ADL executed in parallel. The memory function of the psychological model allows us to detect nested ADL, because previous sensor events still have an influence on the current results. The evaluation was done with a publicly available dataset and benchmark [8], which includes three different datasets form different locations, sensor sets and ADL. The accuracy of the introduced and unsupervised approach was $97 \%$ for all three datasets where the best benchmark's result using conditional random fields achieved $92 \%$. The better result was achieved by neglecting the problems of input sequences, detecting parallel ADL and the probability distribution function instead of one harsh decision where the sensor events did not provide enough evidence to choose the one and only ADL. 
Acknowledgments: This research was part of the context of the European TRIBUTE project (FP7 Contract No. NMP2-LA-2013-608790) and the German Project AUTAGEF: Automatische Assistenz in Gefahrensituationen (Project No. 16SV4012).

Author Contributions: Jana Clement has done the scientific research, conceived of, designed and performed the experiments, analyzed the data and wrote the paper. Klaus Kabitzsch contributed reagents/materials/analysis tools/sensors and provided the laboratories. All authors have read and approved the final manuscript.

Conflicts of Interest: The authors declare no conflict of interest. The founding sponsors had no role in the design of the study; in the collection, analyses or interpretation of data; in the writing of the manuscript; nor in the decision to publish the results.

\section{References}

1. Clement, J.; Kabitzsch, K. A Combined Stochastic Memory Model for ADL Detection. In Proceedings of the 10th International Conference on PErvasive Technologies Related to Assistive Environments (PETRA 17), Rhodos, Greece, 21-23 June 2017.

2. Harrefors, C.; Saevenstedt, S.; Axelsson, K. Elderly people's perceptions of how they want to be cared for: An interview study with healthy elderly couples in Northern Sweden. Scand. J. Caring Sci. 2009, 23, 353-360. [CrossRef] [PubMed]

3. Lee, V.M.S.; Wong, T.W.; Lau, C.C. Home accidents in elderly patients presenting to an emergency department. J. Accid. Emerg. Nurs. 1999, 7, 96-102. [CrossRef]

4. Deutsches Rotes Kreuz. Every Third Mission Is No Emergency. Press Release of a German Emergency Agency. 2014. Available online: https://www.drk.de/presse/pressemitteilungen/meldung/drk-jederdritte-einsatz-ist-kein-notfall/ (accessed on 28 November 2017).

5. Ahrens, M. Home Smoke Alarms: The Data as Context for Decision. J. Fire Technol. 2008, 44, $313-327$. [CrossRef]

6. Yared, R.; Abdulrazak, B.; Tessier, T.; Mabilleau, P. Cooking risk analysis to enhance safety of elderly people in smart kitchen. In Proceedings of the 8th PErvasive Technologies Related to Assistive Environments (PETRA '15), Corfu, Greece, 1-3 July 2015. [CrossRef]

7. Vennedey, V.; Danner, M.; Evers, S.; Fauser, S.; Stock, S.; Dirksen, C.; Hiligsmann, M. Using qualitative research to facilitate the interpretation of quantitative results from a discrete choice experiment: Insights from a survey in elderly ophthalmologic patients. Patients Preference Adherence 2016, 10, 993-1002. [CrossRef] [PubMed]

8. Van Kasteren, T.; Englebienne, G.; Kroese, B. Human Activity Recognition from Wireless Sensor Network Data: Benchmark and Software. Activ. Recognit. Pervasive Intell. Environ. 2011, 4, 165-186.

9. Alcala, J.; Urena, J.; Hernandez, A. Activity Supervision Tool using Non-Intrusive Load Monitoring Systems. In Proceedings of the 20th Conference on Emerging Technologies \& Factory Automation (ETFA), Luxembourg, UK, 8-11 September 2015. [CrossRef]

10. Alemdar, H.; Ertan, H.; Incel, O.; Ersoy, C. ARAS human activity datasets in multiple homes with multiple residents. In Proceedings of the 7th Pervasive Healt, Venice, Italy, 5-8 May 2013; pp. 232-235. [CrossRef]

11. Schmidt, F.; Kabitzsch, K.; Clement, J.; Hildebrandt, D.; Richter, K.-P.; Mueller, G.; Hirschfeld, D. Automated Assistance in Emergency Situations the AUTAGEF Project and its Business Model. In Proceedings of the 4th German AAL-Congress, Berlin, Germany, 25-26 January 2011.

12. European Project TRIBUTE (FP7 Contract Number NMP2-LA-2013-608790). Available online: http:/ / www. tribute-fp7.eu/ (accessed on 28 November 2017).

13. Du, L.; Yang, Y.; He, D.; Harley, R.G.; Habetler, T.G.; Lu, B. Support Vector Machine Based Methods for Non-intrusive Identification of Miscellaneous Electric Loads. In Proceedings of the 38th Annual Conference of IEEE Industrial Electronics (IECON 2012), Montreal, QC, Canada, 25-28 October 2012. [CrossRef]

14. Tapia, E.; Intille, S.; Larson, K. Activity Recognition in the Home Using Simple and Ubiquitous Sensors. In Proceedings of the Second International Conference PERVASIVE 2004, Linz/Vienna, Austria, 21-23 April 2004; pp. 158-175.

15. Noury, N.; Quach, K.A.; Berenguer, M.; Teyssier, H.; Bouzid, M.-J.; Goldstein, L.; Giordani, M. Remote Follow Up of Health Through the Monitoring of Electrical Activities on the Residential Power Line Preliminary Results of an Experimentation. In Proceedings of the 11th International Conference on e-Health Networking, Application \& Services (Healthcom 2009), Sydney, Australia, 16-18 December 2009; pp. 9-13. [CrossRef] 
16. Benmansour, A.; Bouchachia, A.; Feham, M. Human Activity Recognition in Pervasive Single Resident Smart Homes: State of Art. In Proceedings of the 12th International Symposium on Programming and Systems (ISPS 2015), Algiers, Algeria, 28-30 April 2015. [CrossRef]

17. Clement, J.; Ploennigs, J.; Kabitzsch, K. Enhanced Inactivity Diagram to Meet Elderly Needs. In Proceedings of the Partnerships for social innovation in Europe (AAL-Forum), Italy, Lecce, 26-28 September 2011; pp. 217-225.

18. Noury, N.; Berenguer, M.; Teyssier, M.; Bouzid, M.-J.; Giordani, M. Building an Index of Activity of Inhabitants from Their Activity on the Residential Electrical Power Line. IEEE Trans. Inf. Technol. Biomed. 2011, 15, 758-766. [CrossRef] [PubMed]

19. Yala, N.; Fergani, B.; Fleury, A. Feature Extraction for Human Activity Recognition on Streaming Data. In Proceedings of the Inist Presentation at LIBER 2015 (INIST), Madrid, Spain, 2-4 September 2015. [CrossRef]

20. Ye, C.; Xia, Y.; Sun, Y.; Wang, S.; Yan, H.; Mehmood, R. ERAR: An Event-driven Approach for Real-time Activity Recognition. In Proceedings of the International Conference on Identification, Information \& Knowledge in the Internet of Things (IIKI), BeiJing, China, 22-23 October 2015. [CrossRef]

21. Fernandez-Llata, C.; Garcia-Gomez, J.M.; Vicente, J.; Naranjo, J.C.; Robles, M.; Benedi, J.M.; Traver, V. Behavior Patterns Detection for Persuasive Design in Nursing Homes to Help Dementia Patients. In Proceedings of the 33rd Annual International Conference of the IEEE Engineering in Medicine and Biology Society (EMBC 2011), Boston, MA, USA, 30 August-3 September 2011. [CrossRef]

22. Ye, J.; Stevenson, G.; Dobson, S. USMART: An unsupervised semantic mining activity recognition technique. ACM Trans. Int. Intell. Syst. 2015, 4, 16. [CrossRef]

23. Clement, J.; Ploennigs, J.; Kabitzsch, K. Smart Meter: Detect and Individualize ADL. In Proceedings of the 5th Deutscher AAL-Kongress 2012, Berlin, Germany, 21-22 January 2012; pp. 107-122.

24. Ebbinghaus, H. Ueber Das Gedaechtnis. Untersuchungen Zur Experimentellen Psychologie; Wissenschaftliche Buchgesellschaft: Darmstadt, Germany, 1992; ISBN 9783534052554.

(C) 2017 by the authors. Licensee MDPI, Basel, Switzerland. This article is an open access article distributed under the terms and conditions of the Creative Commons Attribution (CC BY) license (http:/ / creativecommons.org/licenses/by/4.0/). 